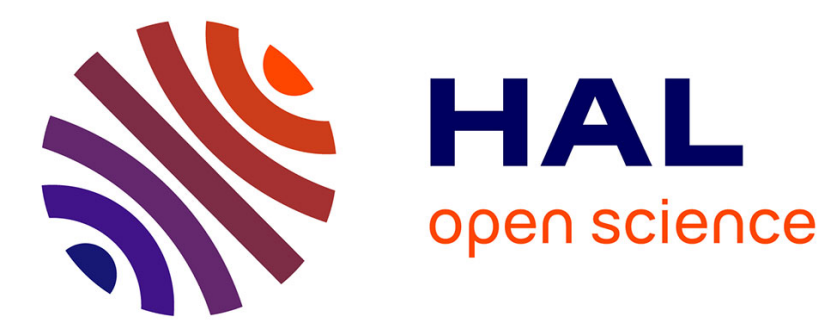

\title{
Une expérience d'élagage mécanique
}

Guy de La Fouchardière

\section{To cite this version:}

Guy de La Fouchardière. Une expérience d'élagage mécanique. Revue forestière française, 1968, 7-8, pp.497-500. 10.4267/2042/24965 . hal-03389831

\section{HAL Id: hal-03389831 \\ https://hal.science/hal-03389831}

Submitted on 21 Oct 2021

HAL is a multi-disciplinary open access archive for the deposit and dissemination of scientific research documents, whether they are published or not. The documents may come from teaching and research institutions in France or abroad, or from public or private research centers.
L'archive ouverte pluridisciplinaire HAL, est destinée au dépôt et à la diffusion de documents scientifiques de niveau recherche, publiés ou non, émanant des établissements d'enseignement et de recherche français ou étrangers, des laboratoires publics ou privés. 


\title{
UNE EXPÉRIENCE D'ÉLAGAGE MECANIQUE
}

\author{
PAR \\ G. de la FOUCHARDIËRE \\ Ingénieur en Chef GREF - D.D.A. de Saint-Brieuc
}

Un certain nombre de propriétaires forestiers des Côtes-du-Nord se sont réunis en CUMA pour l'achat et l'utilisation dans le reboisement du ( Singe ), c'est-à-dire de l'élagueuse mécanique Fichtel et Sachs, matériel qui les avait séduit au cours d'une démonstration.

Cet engin fonctionne depuis un an et l'on a déjà pu tirer des enseignements très'intéressants sur son fonctionnement et son prix de revient, c'est pourquoi il me semble bon de faire le point de cette expérience qui pourra servir à d'autres.

L'engin coûte environ $5000 \mathrm{~F}$, il pèse une cinquantaine de kilos et il faut deux hommes pour le manipuler. Pratiquement, il ne peut être employé que dans des boisements complets, homogènes et âgés d'une vingtaine d'années même lorsqu'il s'agit d'essences à croissance très rapide comme le Douglas ou le Sitka; il faut en effet que les arbres aient déjà perdu leur conicité juvénile pour que l'élagueuse puisse monter à 8 ou 10 mètres, le diamètre minimum étant de $10 \mathrm{~cm}$. Par contre, il ne faut pas que le peuplement soit trop vieux car alors les arbres d'élite, ceux justement que l'on aurait le plus d'intérêt à élaguer, présentent à la base un diamètre supérieur à $23 \mathrm{~cm}$, ce qui constitue le maximum. Le peuplement doit être préparé, c'est-à-dire que pour qu'il n'y ait pas d'empêchement à la circulation et pas de perte de temps, tous les arbres, ou du moins la très grosse majorité d'entre eux, soient déjà élagués à la main sur $2 \mathrm{~m}$ de haut. Cet élagage peut être réduit en ne nettoyant par exemple, mais alors d'une manière complète, qu'un interligne sur 4. Les arbres à élaguer, 4 à 600 à l'hectare, doivent être marqués à l'avance à la peinture. Ils doivent être convenablement choisis, plus d'ailleurs en raison de leurs qualités intrinsèques que de leur espacement, mais cependant il faut que leur répartition soit sensiblement uniforme parce que les arbres élagués seront destinés en principe à être les arbres d'avenirs restant jusqu'à la coupe définitive et au profit desquels les éclaircies seront faites successivement.

Sur un chantier ainsi préparé pour un élagage moyen de $8 \mathrm{~m}$ de haut, le Singe peut travailler entre 30 et 40 arbres à l'heure, 
soit environ 250 dans la journée. Ce chiffre est le résultat moyen obtenu après un an de travail (et non l'extrapolation d'une démonstration).

Le prix de revient est d'environ $1,20 \mathrm{~F}$ par arbre élagué, la maind'œuvre étant de 2 ou 3 hommes dont l'un, toujours le même, est responsable de la machine et l'autre ou les autres des manœuvres fournis par le propriétaire chez qui on procède à l'élagage. Ce prix de $1,20 \mathrm{~F}$ est évidemment très compétitif quand on le compare à l'élalage manuel qui revient au moins au double. Il est d'ailleurs encore au-dessus de la réalité car il est calculé (maind'œuvre, carburant, entretien, amortissement) sur un amortissement au bout de 8000 arbres élagués, or actuellement ce chiffre est déjà dépassé et l'appareil assurera encore, j'espère, un bon service avant de rendre l'âme définitivement.

Techniquement, il y a beaucoup de points de détail qu'il faut préciser. D'abord la mise au point: dès que l'on a commencé à utiliser l'appareil, on s'est aperçu de certaines imperfections auxquelles il a fallu remédier. Les crochets assurant la fermeture de l'appareil étaient trop faibles et il a fallu en faire faire de beaucoup plus solides chez un mécanicien local. La lame, lorsque les branches présentent un gros bourrelet ou ne sont pas parfaitement rectilignes, risque de faire des plaies et l'on a imaginé de mettre un bouton de caoutchouc maintenant cette lame à un centimètre du tronc quelle que soit l'inclinaison de l'appareil. L'usage de l'outil demande également une certaine technique; lorsque l'appareil bute, il est prévu une perche pour l'aider, or, instinctivement, les ouvriers poussaient l'appareil pour le faire monter. Ils se sont aperçus très vite qu'il fallait au contraire tirer l'appareil pour le faire redescendre de quelques centimètres et alors il reprenait sa course normalement. Lorsque l'outil descend, il représente une certaine force vive et l'arrêter en marche avant qu'il ne touche le sol est un exercice qui demande une certaine dextérité et peut même présenter quelques risques. Les utilisateurs ont trouvé un moyen beaucoup plus simple pour le freiner, c'est de ramasser une ou deux branches coupées et de les mettre contre le tronc pour freiner la descente en douceur et sans danger.

Je pourrais citer une foule de petits détails dans ce sens qui, au bout d'un entraînement de quelques jours ou de quelques semaines, ont amélioré très sensiblement le rendement. D'autre part, si le travail fait est sensationnel dans certaines conditions, le Singe ne peut travailler dans toutes les circonstances et sur toutes les essences, il faut avant tout des peuplements fermés, bien homogènes, et où les arbres sont rectilignes, cylindriques et sans trop grosses branches. Pratiquement, on ne peut l'utiliser dans les pins qui sont rarement assez droits et dont les branches toujours importantes en Bretagne du fait de la durée de la végétation présentent de très gros bourrelets qui sont blessés. 
Le travail est très bon sur le pectiné mais la lenteur relative de croissance de cette essence rend douteuse la rentabilité de l'élagage d'autant plus que l'élagage naturel est en général satisfaisant. Il ne reste donc que les exotiques à croissance rapide, sapins de Vancouver, Douglas, Sitka et mélèzes. Pour le sapin géant, il y a un gros inconvénient présenté par les poches de résine de l'écorce qui éclatent sous la pression des pneus et engluent ceux-ci, les faisant déraper. L'on pourrait peut-être y remédier en munissant les pneus moteurs de pointes d'acier type anti-verglas et suffisamment courtes pour ne pas risquer de faire de lésions dans le bois. Pour le Douglas, cet inconvénient se retrouve aussi mais à un degré bien moindre et l'élagage n'en souffre pas. Le mélèze s'élage parfaitement et d'autant mieux que pratiquement il n'y a que des branches mortes à couper, mais très souvent les mélèzes (du Japon surtout) ne sont pas assez rectilignes pour que l'outil puisse être utilisé et c'est pratiquement le Sitka dont il existe des repeuplements très importants sur lequel l'on a opéré dans la très grande majorité des cas (le Sitka étant toujours supérieur en Bretagne à l'épicéa commun, il n'y a pratiquement pas de plantations de cette dernière essence) or, mềme sur le Sitka qui présente toujours les qualités voulues, l'on ne peut travailler que par temps sec sous peine de voir les pneus déraper et là aussi l'adjonction de quelques clous anti-verglas serait souhaitable. Il serait également intéressant d'apporter une petite modification, qui est d'ailleurs prévue, pour que le diamètre maximum puisse passer de 23 à 27 ou $30 \mathrm{~cm}$ car un Sitka de 20 ans faisant $18 \mathrm{~m}$ de haut et élagable jusqu'à $10 \mathrm{~m}$ fait très souvent $25 \mathrm{~cm}$ ou plus à la base où naissent rapidement des contreforts importants et si l'on est obligé de hisser l'appareil à bras jusqu'à $1,50 \mathrm{~m}$ de hauteur, cela représente un effort qui diminue beaucoup le rendement journalier.

Reste la question rentabilité, il ne faut pas perdre de vue que même si l'élagage sur $8 \mathrm{~m}$ de haut ne coûte que $1,20 \mathrm{~F}$, le bénéfice se fera ressentir seulement de nombreuses années après et si l'on comptabilise ces $1,20 \mathrm{~F}$, intérêts compris, même avec un taux assez faible, il est évident qu'au bout de 30 ans ils auront " fait des petits ». Le bénéfice sera la différence du prix de vente entre un arbre non élagué présentant sur toute sa hauteur des nœuds, blancs ou noirs, et l'arbre qui possédera sur $8 \mathrm{~m}$ une bille de pied où, seul, le cour sera noueux, les " rives ) parfaitement propres donnant des bois de premier choix. Dans l'optique des forêts particulières où l'on ne peut évidemment consentir le sacrifice financier nécessité pour la production de bois de haute qualité, à accroissements minces et réguliers, est-ce que les arbres sans nœud mais présentant des cernes de $1 \mathrm{~cm}$ de largeur seront cotés sur le marché à un prix suffisant?.. Il semble bien difficile de répondre à cette question. De toutes façons, il semble être aberrant de provoquer l'élagage total sur tous les arbres d'un peuplement dont certains seront exploités quelques 
années seulement après. Peut-être que non, si ces éclaircies doivent se faire en poteaux de lignes, dont la présentation sera absolument impeccable, mais d'une part l'on n'utilise dans la région en poteaux de lignes que les arbres à duramen différencié (mélèze, pins et Douglas) et avec un minimum de cernes au fin bout qui élimine les plantations ayant poussé trop vite, d'autre part pour les petits bois de sciage il est évident que, trop minces, des bois de rives n'apporteront pas une plus-value suffisante et je suis persuadé que la meilleure technique serait de se contenter d'élaguer 4 à 600 pieds à l'hectare représentant uniquement ce qui sera enlevé à la coupe définitive lorsque les arbres auront environ 50,60 ou 70 de diamètre car les belles billes de pied absolument nettes 'ne manqueront certainement pas de tenter les acheteurs. 